\title{
New Approach to Hot Electron Effects in Si-MOSFETs Based on an Evolutionary Algorithm Using a Monte Carlo Like Mutation Operator
}

\author{
J. JAKUMEIT ${ }^{\mathrm{a}}$, U. RAVAIOLI ${ }^{\mathrm{b}}$ and K. HESS ${ }^{\mathrm{b}}$ \\ a II. Phys. Inst., Uni Köln, Zülpicher Str. 77, D-50937 Köln , Germany, ${ }^{\mathrm{b} B e c k m a n}$ Institute, University of Illinois, Urbana, IL 61801
}

\begin{abstract}
We introduce a new approach to hot electron effects in Si-MOSFETs, based on a mixture of evolutionary optimization algorithms and Monte Carlo technique. The Evolutionary Algorithm searchs for electron distributions which fit a given goal, for example a measured substrate current and in this way can calculate backwards electron distributions from measurement results. The search of the Evolutionary Algorithm is directed toward physically correct distributions by help of a Monte Carlo like mutation operator. Results for bulk-Si demonstrate the correctness of the physical model in the Monte Carlo like mutation operator and the backward calculation ability of the Evolutionary Algorithm. First results for Si-MOSFETs are qualitatively comparable to results of a Full Band Monte Carlo simulation.
\end{abstract}

Keywords: silicon, hot electrons, simulation, evolutionary algorithm, genetic algorithm, Monte-Carlo

\section{SIMULATION APPROACH}

We describe a new method for the calculation of hot electron effects in semiconductors involving extremely high energies above the conduction band edge. The goal is to develop an algorithm for the investigation of hot electron effects in Si-MOSFETs, which is less complex and computational expensive than a full band Monte Carlo program, yet gives more precise information than lucky electron or electron temperature models and can thus be used as a mediator between precise theory and experimental results. To achieve this goal an Evolutionary Algorithm with a mutation operator based on the Monte Carlo technique (MC) is used to search for electron distributions which fit a given substrate current.

The idea of Evolutionary Algorithms (EA) is inspired by the evolution processes that take place in nature, where successful species must adapt continuously to the changing environment by creating new individuals and by selection of the fittest $[1,2]$. This natural optimization technique can easily be transfered to mathematical optimization problems. The objective function $F$ on a search space $S$ can be interpreted as the environment and each vector $\mathrm{v} \in S$ is a possible individual in this environment. The fitness of these mathematical individuals is given by the value of the objective function $F(v)$. The EA searches for the optimal solution, by starting with an initial set of vectors called "population". In each following iteration (generation) a new set of vectors is created from the old population by modifying the fittest of the old vectors. For the new population of the next generation the fittest among the newly created and old vectors are selected. 
To make use of EAs in the investigation of electron distributions in silicon, the calculation of the distribution must be transformed into an optimization problem [3]. In the case presented here, we search for electron distributions $f d(E, x)(f d(E, x)=$ distribution function $f(E, x)$ times density of state $D(E)$ ) which give a defined substrate current $I_{\text {sub }}^{\text {goal }}$, i.e. the search space $S$ is represented by all possible electron distributions $(\mathrm{v} \simeq f d)$ and the objective function is

$$
\begin{aligned}
& F(f d(E, x))=-\frac{\left|I_{\text {sub }}^{\text {goal }}-I_{\text {sub }}^{\text {calc }}\right|}{I_{\text {sub }}^{\text {gal }}} \\
& \text { with } \quad I_{\text {sub }}^{\text {calc }} \sim e \cdot \int f d(E, x) W_{i i}(E) d E d x,
\end{aligned}
$$

where $W_{i i}(E)$ is the impact ionization rate and e the electron charge. For our given problem, the creation of new individuals by modifying, rather than replacing, a previous set is very important. In the problem of finding the 'right' electron distribution, many possible distributions (even very unphysical ones) may correspond to the same substrate current $I_{s u b}^{g o a l}$. Therefore, a physical mutation operator is used to lead the search of the EA to the physically meaningful electron distributions. This mutation operator is based on a MC-like process and will be discussed in detail below. Besides this physical mutation operator, the electron distributions are modified by two random processes. The first increases or decreases the tail of the distribution while keeping the value at $E=0$ fixed. The second changes the distribution around a randomly chosen point $E_{i}$ smoothly. The magnitude of the change in both operators is again chosen randomly but never to exceed $10 \%$. The selection of the new population from the old and the newly greated vectors is performed by a Threshold-Acceptance selection method. The EA stops when a solution is found with a relativ difference between $I_{\text {sub }}^{\text {calc }}$ and I goal less than 0.001 or the number of generations exceeds 1000.

The MC-like mutation of the electron distribution by the physical mutation operator can be performed by transfering a part of the distribution around a randomly chosen energy $E_{\text {start }}$ (and place $x_{\text {start }}$ ) into a MC-electron, simulating the drift and scattering of the electron in the same way as in a standard MC-simula- tion to a final energy $E_{\text {final }}\left(\right.$ and place $\left.x_{\text {final }}\right)$ and returning the electron density represented by the MCelectron to the distribution. For the MC-simulation of the electron movement a simple one-valley effective mass approach is used, taking the effective mass $m_{\text {eff }}$ as a free parameter. Scatterings due to phonon absorption, emission and impact ionization are included in the model. The phonon scattering rates are calculated by a full band approach and the impact ionization rate computed in [4] was taken (assuming zero field and no collision broadening). The phonon scattering and impact ionization rates are then multiplied by factors $f_{\text {phonon }}$ and $f_{\text {impact }}$, respectively. The introduction of these two parameters gives us more flexibilty to calibrate the simple model by comparison with full band approaches.

\section{RESULTS AND DISCUSSION}

The ability of the physical mutation operator to lead the search for a distribution function towards physically correct ones can be tested by setting the population size of the EA to 1 , the mutation and recombination probability of all nonphysical operators to zero and the threshold for the selection of the new population to a very high value, so that the new individual is always selected. In this way all optimization is suppressed and the effect of the physical mutation operator on the distribution function can be investigated separately. Using this parameter set the Evolutionary Algorithm becomes a new type of Monte-Carlo technique, which we will refer to as the Mutation Operator Monte Carlo (MOMC) method.

In fig. 1 the electron distributions obtained by the MOMC-technique (dashed lines) and the EA (dotted line) are compared with results of a Full Band Monte Carlo (FBMC) program with statistical enhancement of the high energy tail (solid line) for bulk silicon. In the MOMC and EA the parameter set $m_{\text {eff }}=1.0$, $f_{\text {phonon }}=1.0$ and $f_{\text {impact }}=1.6$ was used. For different electric fields in the range $1 \mathrm{kV} / \mathrm{cm}$ to $500 \mathrm{kV} / \mathrm{cm}$ the results of MOMC agree well with the results of the FBMC over almost the whole energy range. The results of the EA are almost identical to those of the 


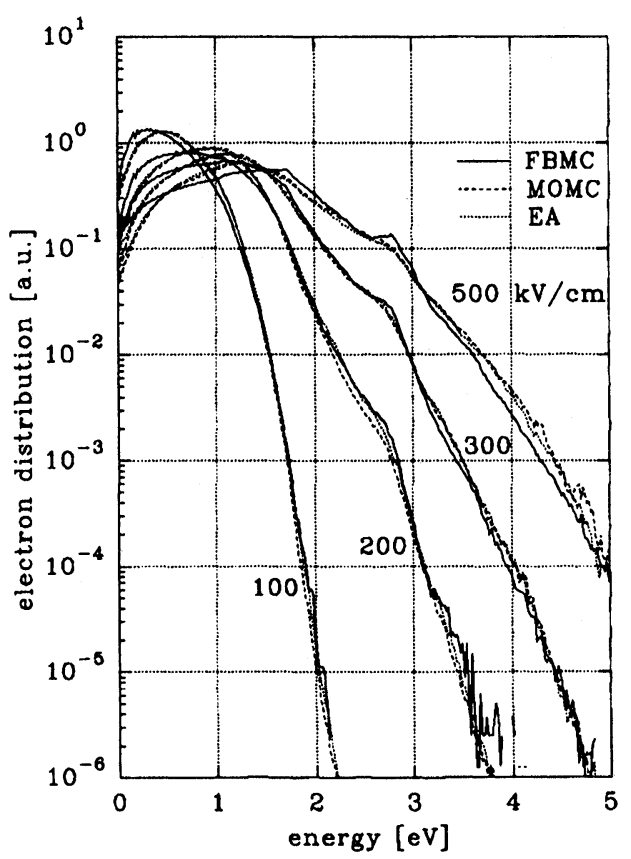

FIGURE 1 Electron distributions given by the FBMC (solid), MOMC (dashed) and EA (dotted) for $F=100,200,300$ and $500 \mathrm{kV} / \mathrm{cm}$

MOMC. Thus for this given problem, backward calculations with the EA do not improve the results, simply because the physical mutation operator itself is so powerful. The simple model using an effective mass approach with three well chosen scattering rates contains most of the information necessary to simulate the electron distribution of electrons in bulk silicon over the whole energy range. Compared with the FBMC the MOMC has the advantage of a better resolution of the high energy tail and a about ten times higher computation speed.

For simulations when the physical mutation operator is less well known (e.g. complex device geometries) the addition of EA should be beneficial. To demonstrate this we have changed the factor $f_{\text {phonon }}$ which adjusts the strength of the phonon scattering rates away from its optimum value. Fig. 2 shows the results obtained by the MOMC-technique (long dashed) and the EA (dotted) for $f_{\text {phonon }}=0.8$ at an electric field of $300 \mathrm{kV} / \mathrm{cm}$ and compares them with the result of the FBMC. As expected, this physi-

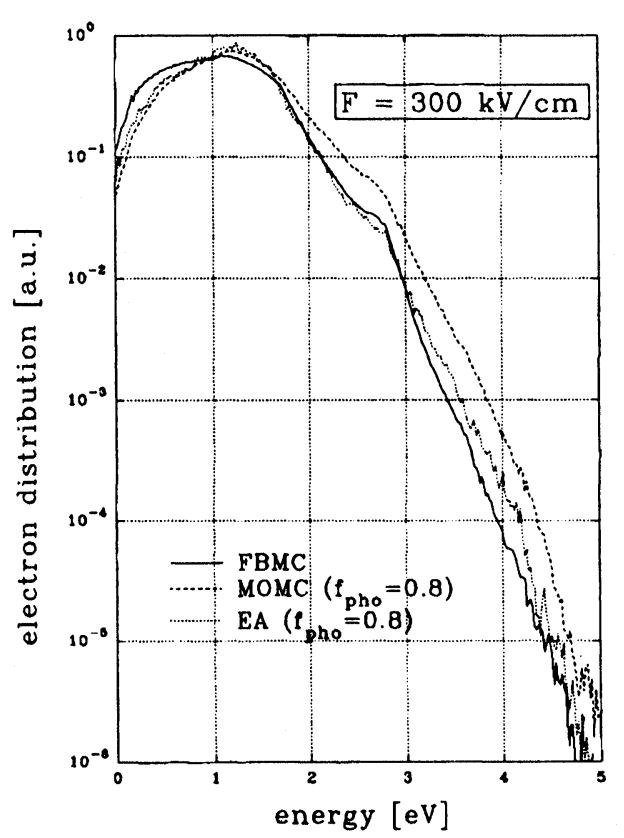

FIGURE 2 Results for the MOMC (dashed) and EA (dotted) for a not optimal parameter $f_{\text {phonon }}=0.8$

cal mutation operator leads to a higher tail of the distribution because of the reduced scattering. However, the EA, aiming at the substrate current, calculated from the FBMC-result, gives still an electron distribution fairly close to the correct distribution. Thus even with a slightly wrong physical model the EA still finds appropriate solutions due to its ability of backward calculation.

For the simulation of a Si-MOSFET the spatial dependence of the carrier density $n(x)$, the electric field $F(x)$ and the potential $V(x)$ are needed as input for the MOMC and EA. They were obtained by a comercial drift-diffusion-program (ISE-software). Fig. 3 shows the electron distribution (a) and $I_{s u b}(E, x)$ (b) for a $0.8 \mu \mathrm{m} \mathrm{Si-MOSFET} \mathrm{with} V_{d}=6 \mathrm{~V}$ and $V_{g}=7 \mathrm{~V}$ obtained by the EA. The electron distribution shows the heating of the electron gas by the high fields before the drain $(x<1.0 \mu \mathrm{m})$ and the cooling in the drain. The substrate current peaks shortly before the drain at an energy around $2 \mathrm{eV}$. Both results are qualitatively comparable with those of a FBMC. 

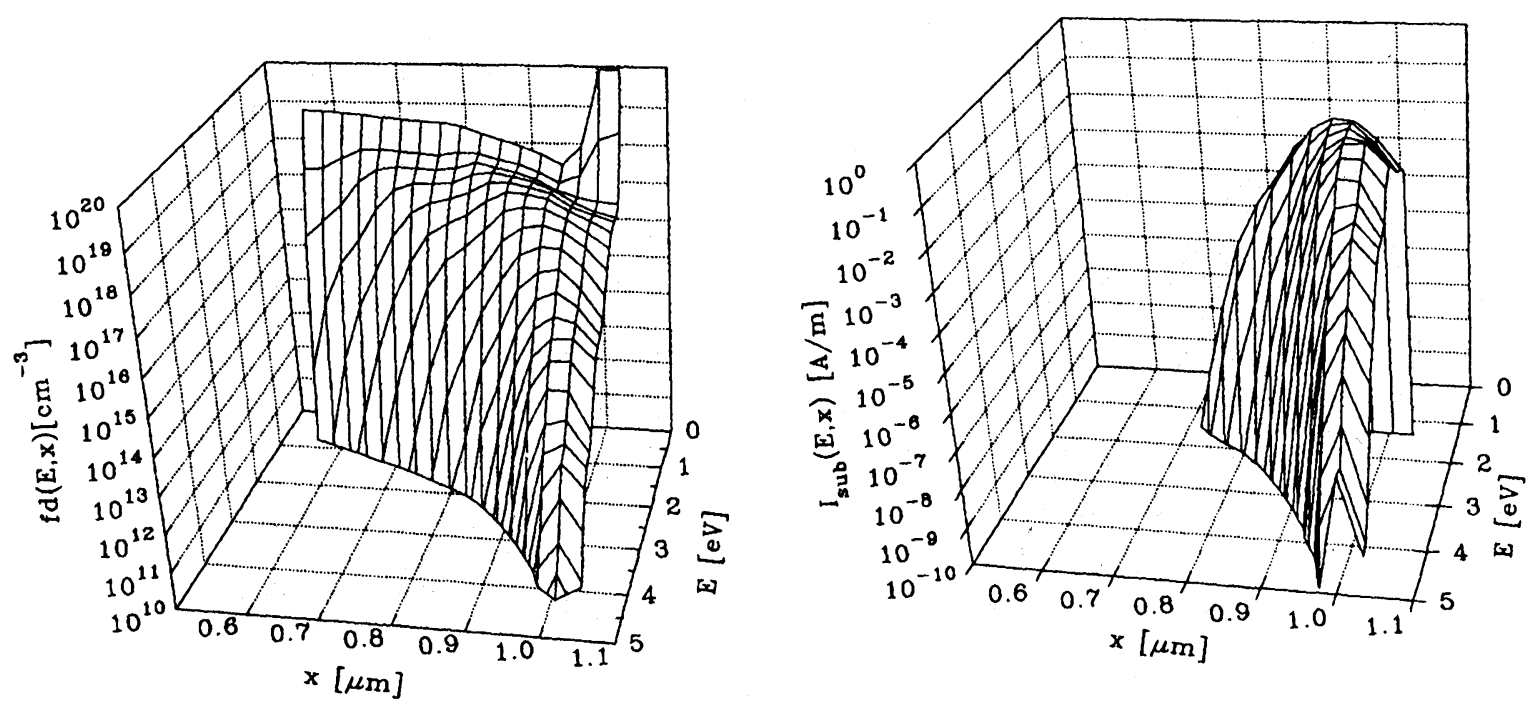

FIGURE 3 Results for a $0.8 \mathrm{Si}$-MOSFET. Electron distribution (a) and substrate current (b) as function of $\mathrm{E}$ and $\mathrm{x}$. The drain begins at $\mathrm{x}=1.0 \mu \mathrm{m}$

\section{CONCLUSION}

A new approach to hot electron effects in silicon was introduced, based on an Evolutionary Algorithm using a Monte Carlo like physical mutation operator. This physical mutation operator was proven to be powerful enough to describe the energy distribution of electrons in the conduction band of bulk silicon over the whole energy range for various electric fields, leading to a new type of Monte Carlo technique, which we called Mutation Operator Monte Carlo simulation. Compared to FBMC simulation, the MOMC gives a better resolution of the high energy tail and is less computational expensive. Concerning the Evolutionary Algorithm we have demonstrated that this approach yields correct electron distributions even with a slightly incorrect or oversimplified physical model due to the backward calculation ability. First results with this new approach for a $0.8 \mu \mathrm{m} \mathrm{Si}$ MOSFET indicate that the Evolutionary Algorithm is a promising tool to investigate device reliability related hot electron effects.

\section{References}

[1] D. E. Goldberg, Genetic algorithms in Search, Optimization and Machine Learning, Addison-Wesley, 1989

[2] Z. Michalowicz, Genetic algorithms + Data structure $=$ Evolution Programs, Springer-Verlag, New York 1994

[3] J. Jakumeit, Appl. Phys. Lett. 66, 1995, p. 1812

[4] J. Bude, K. Hess, G. J. Iafrate, Phys. Rev. B 45, 1992, p. 10958

\section{Biographies}

Jürgen Jakumeit studied physics at the University of Cologne and finished his dissertation in 1994. His research concerned quantum size effects in semiconductor structures. During two half year stays at the University of Michigan, Ann Arbor, MI and the University of Illinois, Urbana-Champaign, IL, he got engaged in the field of device simulation. At the moment he works as Postdoc at the University of Cologne on the simulation of silicon MOSFETs and the investigation of quantum interference effects.

Umberto Ravaioli is a Professor in the Department of Electrical and Computer Engineering of the University of Illinois at Urbana-Champaign. His main research interests are in the areas of semiconductor 
device physics and simulation, numerical methods, and high performance computing.

Karl Hess is a Professor in the Department of Electrical and Computer Engineering of the Univer- sity of Illinois at Urbana-Champaign. His main research interests are in the areas of semiconductor device physics and simulation, numerical methods, and high performance computing. 

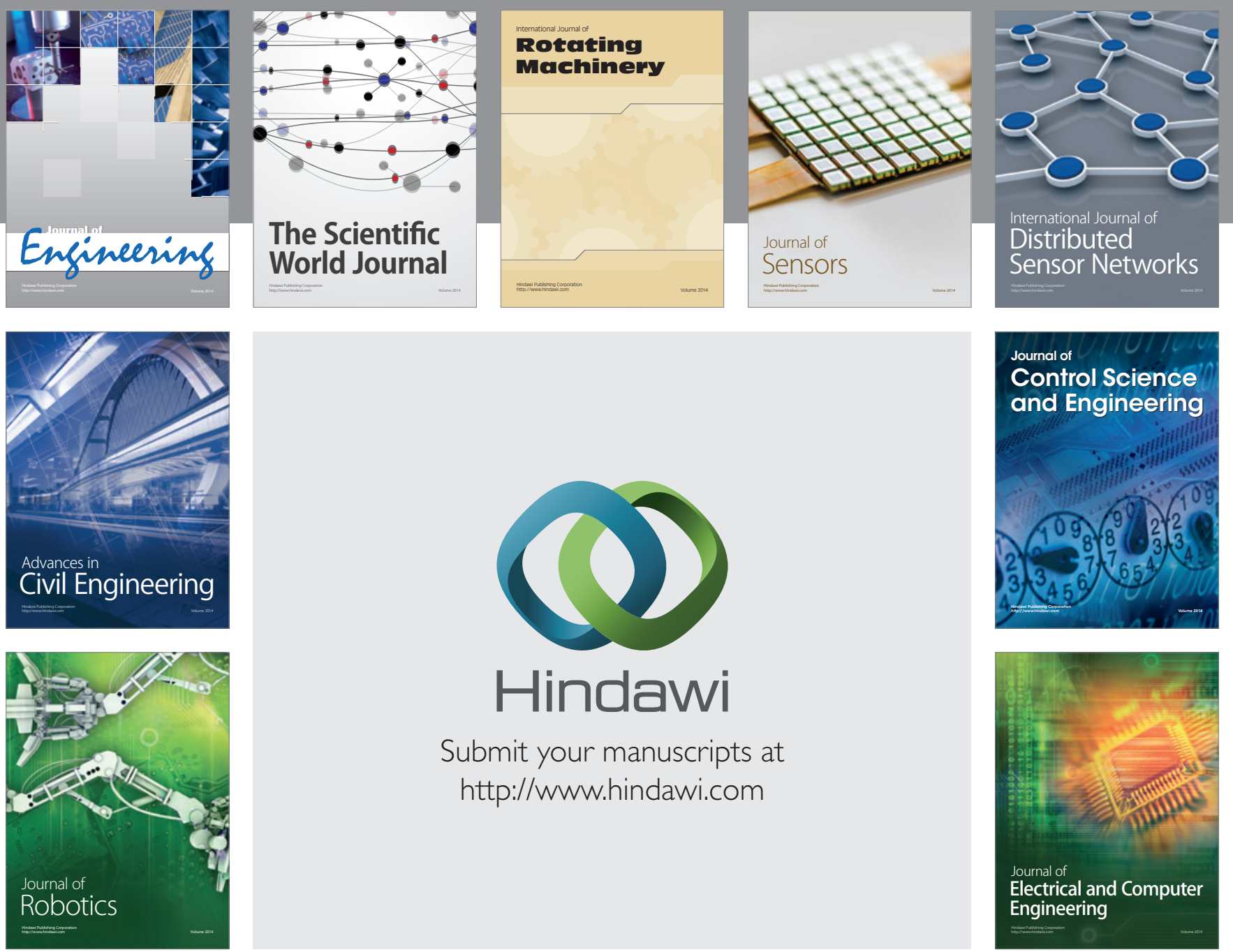

Submit your manuscripts at

http://www.hindawi.com
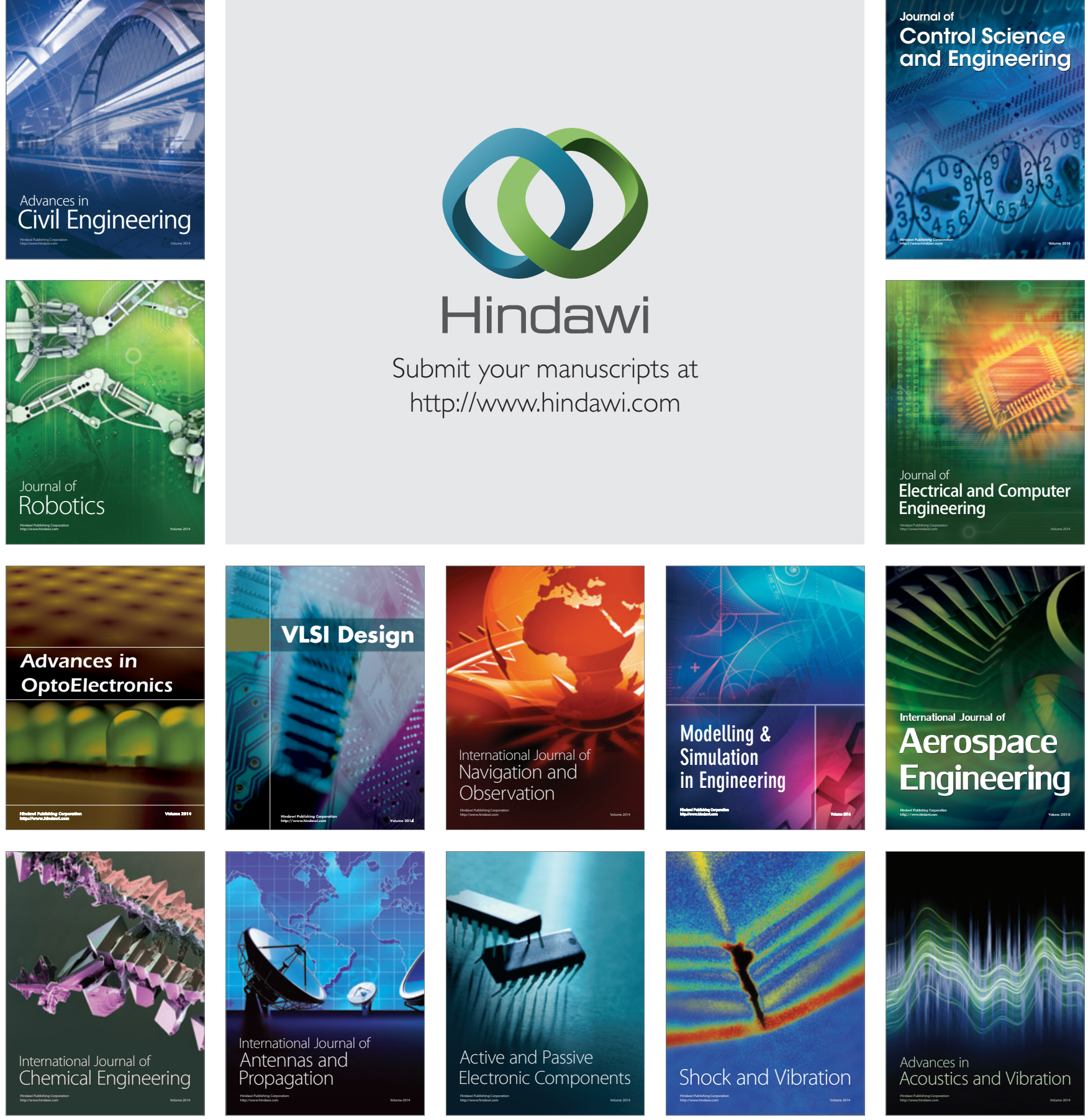